\title{
CLOCK TECHNOLOGY DEVELOPMENT FOR THE LASER COOLING AND ATOMIC PHYSICS (LCAP) PROGRAM
}

\author{
W.M. Klipstein, R.J. Thompson, D.J. Seidel, J. Kohel, and L. Maleki, Jet Propulsion Laboratory, California \\ Institute of Technology, Pasadena, California, 91109
}

Mailing address: Bill Klipstein, MS 298/100, Jet Propulsion Laboratory, 4800 Oak Grove Drive,
Pasadena, California 91109, U.S.A.

\begin{abstract}
The Time and Frequency Sciences and Technology Group at Jet Propulsion Laboratory (JPL) has developed a laser cooling capability for flight and has been selected by NASA to support the Laser-Cooling and Atomic Physics (LCAP) program. Current work in the group includes design and development for the two laser-cooled atomic clock experiments which have been selected for flight on the International Space Station
\end{abstract}

\section{INTRODUCTION}

The Time and Frequency Sciences and Technology group at the Jet Propulsion Laboratory (JPL) has been developing a laser-cooling capability for flight projects in the Laser Cooling and Atomic Physics (program). Two clock experiments have been selected by NASA for flight aboard the International Space Station (ISS): the Primary Atomic Reference Clock in Space (PARCS), with principle investigators at the National Institute of Standards and Technology (NIST) and the University of Colorado; and the Rubidium Atomic Clock Experiment (RACE), with principle investigator at Yale University.

NASA's Fundamental Physics program has identified laser cooling as one of three disciplines which are poised to take advantage of the microgravity environment offered by the International Space Station (ISS) and the Space Shuttle. In addition, 11 groundbased investigations have been funded to date on topics including atomic clocks, Bose-Einstein Condensation (BEC), Electric Dipole Moment (EDM) searches, and atom interferometry

The two flight projects are both represented at this meeting,' so this paper will not cover specifics of those missions except as they pertain to our role at JPL.

* This work was performed at the Jet Propulsion Laboratory, California Institute of Technology under a contract with the National Aeronautics and Space Administration.

\section{DESIGN, BUILD, TEST, AND INTEGRATE}

Our group will support LCAP missions through the design, construction and integration of instruments capable of meeting the science goals within the constraints placed upon flight hardware and available platforms on the ISS. Design and development work is aided by ground testbeds in our laboratory, drawing as well on the diverse experience of our partners at NIST, Yale, and the University of Colorado. Of particular use to the current flight programs we have extensive clocktesting capabilities, environmental test chambers and a miniature shake table which allow us to do preliminary component testing as shown in Fig. 1. More thorough testing of the integrated apparati will use the JPL flight hardware testing facility.

We have identified three issues which must be resolved early on for PARCS specifically: design of shutters for light baffling, a viable and flexible laser system, and the capability to transfer the clock frequency from the ISS down to the ground via GPS Carrier Phase. Shutter

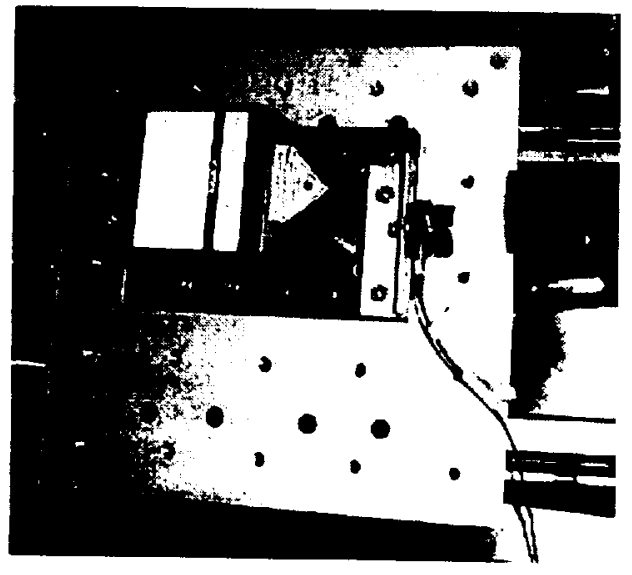

Figure 1 New Focus Vortex laser on miniature shake table. The laser performed well in our tests. 
concepts are being explored with the help of mechanical designers at JPL.

\section{LASERS}

For a laser system we are evaluating an array of optical components for flight worthiness. For the lasers themselves, we are considering different arrangements of narrow-frequency master lasers followed by optical power amplifiers. Current promising options for the master laser include extended cavity diode lasers and distributed Bragg reflector (DBR) laser diodes. We have performed preliminary vibration testing on a New Focus Vortex extended cavity laser on our in-house shake table, up to the level required for launch at low frequencies, and monitored the power during shaking. The laser did not stop lasing, nor suffered any obvious power fluctuations. After shaking the laser was right on the atomic resonance, so we feel we have an outstanding starting point for using this as a master laser. The only disadvantage comes in size as compared to a DBR laser. For DBR master, we have been planning on using a Yokogawa DBR, which has a specified output power of only $5 \mathrm{~mW}$ but have been run at $10 \mathrm{~mW} .^{2}$ The broader linewidth of this laser should not impact the performance of PARCS, so the small package and simple electronics are quite attractive. The problem lies in availability, as Yokogawa may discontinue selling these diodes commercially.

For power amplifiers, nothing currently comes close to SDL tapered amplifiers with an output power in excess of $500 \mathrm{~mW}$. Quality control on the optical coatings and longevity of these devices remain a concern. Injectionlocked Fabry-Perot lasers remain an attractive alternative, but for high-power applications become unwieldy in number.

\section{GPS Carrier Phase Frequency Transfer}

In order to evaluate the performance of clocks in orbit and realize their full science return, the frequencies of the clocks must be relayed to the ground. The most promising candidate for this transfer is GPS Carrier Phase, in which the pseudorange date and carrier data from multiple satellites is used to provide extremely precise position and clock-rate information. " The study of GPS Carrier Phase for PARCS is mainly being carried out by the Orbiter and Radio Metric Systems Group and the GPS Systems Group at JPL. In order to meet the science goals of PARCS, position uncertainties must be below $10 \mathrm{~cm}$. velocity uncertainties need to be below $1 \mathrm{~cm} / \mathrm{s}$, and time transfer uncertainties need to be below 100 ps.

These demanding requirements are consistent with the capabilities of GPS Carrier Phase provided platform- dependent difficulties can be overcome. For example, simulations assume 12 GPS satellites in view. ${ }^{4}$ The existing ISS GPS antennae see between 3-6 satellites, with viewing obscured by ISS structures. These antennae are also only single-frequency devices, while GPS Carrier Phase requires both the $\mathrm{L} 1$ and L2 band signals to correct for atmospheric delays. Custom antennae will need to be deployed and the signals will need to be brought from the exterior of the station to the interior location of the clocks using high-quality RF or optical fiber links. Currently no appropriate feedthroughs exist, posing a considerable challenge, but some possibility still remains that a change in ISS design can be approved. While this seems unlikely, there is considerable interest at NASA in upgrading the communications capabilities of the ISS by adding antennae feeds and antennae to the top of the Centrifuge Accommodation Module (CAM), the tallest structure on the ISS and the only component whose designs are not yet finalized. The CAM also appears to be prime real estate for antenna placement, but the placement of high-power transmitting antennae at this location may overwhelm the weak GPS signal.

Multipathing of the signals also poses a severe problem for GPS Carrier Phase. Reflections of the GPS signal at the $-70 \mathrm{dBm}$ level $(-20 \mathrm{~dB}$ below the expected signal amplitude) may disturb the Carrier Phase solution. The ISS is a complicated mechanical structure, and to better understand the impact of multipath we have assembled a scale model purchased
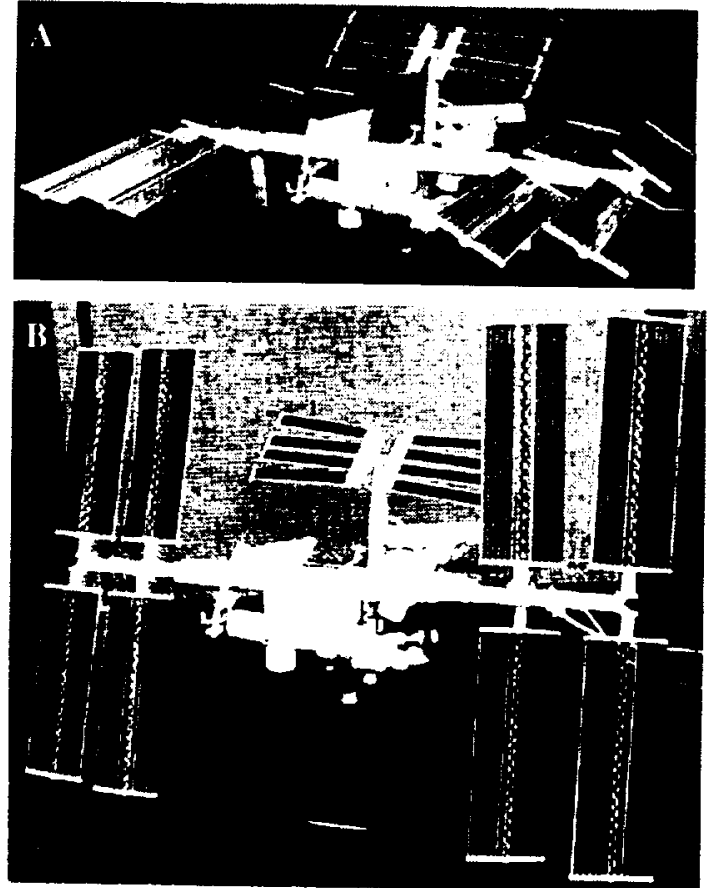

Figure 2 a) View of ISS with solar arrays in plane; b) View of ISS with solar arrays out of plane. 
commercially. Two pictures of this model are shown below. The first shows the "normal" view of the ISS, in which the solar panels are in the plane of the Station, and the CAM is higher than all points except for the Russian power platform. The second picture shows the view with the solar arrays completely out of the plane as they track the sun. It is clear that no point on the station will be above the moving solar arrays, which obscure viewing and provide many surfaces for multipathing.

\section{AVAILABLE SCIENCE PLATFORMS ON THE ISS}

As missions progress through a series of science reviews, issues of design for space and integration into the Space Shuttle and ISS flight manifests become a growing priority. The Shuttle and ISS have different safety and quality assurance requirements. Vibration testing mainly relates to the launch on the shuttle, while lifetime issues speak mainly to the long duration of Station missions.

The ISS carries a number of different platforms for science payloads, distinguished first and foremost by those interior to the station and those exterior. The various platforms provide different allocations of space, power, and environmental control. For the two flight projects, we are currently baselining the EXPRESS Racks, shown in Fig. 4, utilizing the Active Rack Isolation System (ARIS) inside the U.S. Laboratory Module. Only in the ARIS systems will the Station provide a true microgravity vibratory environment. By carefully reviewing the capabilities available on the various science platforms we are able to design our instruments to operate within the available envelopes, so while launch lies far in the future, these issues must be addressed in the earliest stages of the project.

${ }^{1}$ K. Gibble, "Laser-Cooled Microgravity Clocks," and S. Jefferts, et al., "PARCS: A Primary Atomic Reference Clock in Space," from the proceedings from this conference.

2 John Kitching, private communication.

${ }^{3}$ See, for example, K. Larson and J. Levine, "Timetransfer using the Phase of the GPS Carrier," IEEE

Trans. On Ultrasonics, Ferrooelectronics and

Frequency Control, 45 pp. 539-40, 1998, and

references therein.

${ }^{4}$ Sien Wu, of the Orbiter and Radio Metric Systems

Group at JPL, private communication.

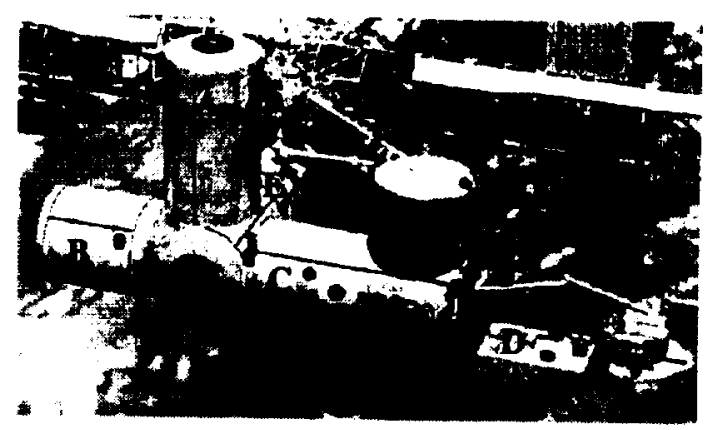

Figure 3 View of the science modules on the ISS: A) Centrifuge Accommodation Module (CAM); B) ESA Science Module; C) Japanese Experiment Module (JEM); D) JEM External Facility; E) US Lab.
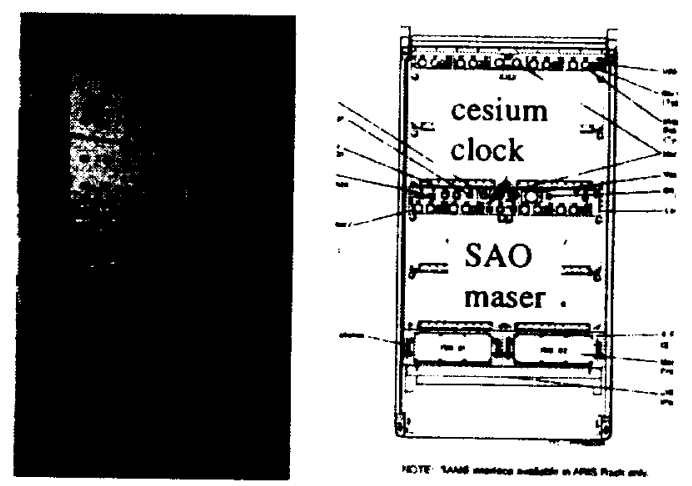

Figure 4. EXPRESS racks incorporating the Active Rack Isolation System (ARIS) look promising for PARCS. One configuration is shown on the right. 